\section{El Estrés Laboral y su Influencia en el Desempeño de los Trabajadores}

\author{
Labor Stress and Its Influence in the Performance \\ of Workers
}

\section{RESUMEN}

El estrés tiene una relación directa con el desempeño laboral, y otras variables más; según las diversas investigaciones realizadas y bases teóricas estudiadas sobre el tema. El estrés laboral es un problema cada vez más frecuente, sin embargo, a pesar de que existen programas para tratarlo y prevenirlo, no ha disminuido, según las estadísticas.

Muchos expertos han tratado de definir estrés laboral, pero el concepto más completo es la Organización Mundial de la Salud (OMS, 2004) que define a esta variable, como: "la respuesta fisiológica, psicológica y de comportamiento de un individuo que intenta adaptarse y ajustarse a presiones internas y externas. El estrés laboral aparece cuando se presenta un desajuste entre la persona, el puesto de trabajo y la propia organización". (p.4). La globalización e inestabilidad por la que atraviesan las organizaciones en estos años, hace que los trabajadores se frustren y con ello se tensionen emocionalmente; y esto a su vez conlleva a un aumento de los niveles de estrés laboral y por ende un bajo desempeño laboral. Por lo mismo, la consideración por investigar la variable estrés laboral y el desempeño ha aumentado hoy en día.

En esta reflexión se ha revisado bastante información sobre esta problemática, para poder ofrecerles un panorama general y actualizado de ambas variables.

Asimismo, mostraremos los principales enfoques, conceptos del estrés laboral y su relación con el desempeño laboral, y cuáles son las causas y algunas propuestas de prevención para su control.

Palabras claves: Estrés; desempeño laboral; organizaciones.

\begin{abstract}
Stress has a direct relationship with work performance, and other variables; according to the diverse investigations carried out and theoretical bases studied on the subject. Work-related stress is an increasingly frequent problem, however, although there are programs to treat and prevent it, it has not diminished, according to statistics.

Many experts have tried to define work stress, but the most complete concept is the World Health Organization (WHO, 2004) that defines a variable,
\end{abstract}

\section{Evelyn Carina Zuñiga Cristobal}

Evelyn_z_c@hotmail.com

Universidad Cayetano Heredia 
such as: "the physiological, psychological and behavioral response of an individual trying to control and adjust to internal and external pressures. Work stress appears when there is a mismatch between the person, the job and the organization itself." (p.4).

The globalization and instability that organizations are going through in these years, makes workers feel frustrated and thereby become emotionally stressed; and this in turn leads to an increase in work stress levels and therefore a low work performance.

For this reason, the evaluation for investigating the variable work stress and performance has increased today. In this reflection, a lot of information on this problem has been reviewed, in order to offer an overview and updated of both variables.

Likewise, we will show the main approaches, concepts of work stress and their relation to work performance, what are the causes and some prevention proposals for their control.

Keywords: Stress; work performance; organizations.

\section{INTRODUCCIÓN}

El interés por estudiar el estrés laboral y como afecta en el desempeño de los trabajadores se ha incrementado notablemente, siendo así un tema común en nuestros días; es un problema muy serio y muy notorio, ya que algunas personas padecen de enfermedades causadas por esta variable.

El estrés laboral no solo afecta la salud de los trabajadores, sino también afecta directamente a la empresa, pues el trabajador baja su productividad, su desempeño laboral y le cuesta relacionarse con los demás, a su vez esto genera mayor ausentismo laboral, descansos médicos, necesidad de reemplazo; en si son muchas las razones que hacen necesario su tratamiento oportuno.

En la actualidad, las organizaciones dependen del capital humano para realizar las tareas cotidianas, por lo mismo que están sujetos a sin números de presiones con la finalidad de poder alcanzar las metas trazadas por la empresa, y sobre todo mantenerse vigentes en el mercado. Otro aspecto muy importante, es que las personas dedican mayor parte de su tiempo en el trabajo, buscando tener un mejor desempeño y sobre todo que su jefe tome en cuenta su esfuerzo; aunque necesariamente no es así, se olvidan de lo más esencial la familia, y el entorno social.

La Asamblea Mundial de la Salud (AMS, 1974) sugirió, "al director general de la Organización Internacional del Trabajo la necesidad de establecer programas multidisciplinares para el análisis e investigación de estas temáticas, así como el desarrollo de medidas para su intervención y prevención en el entorno laboral". Este enfoque suele llamarse 'estudio de estrés laboral' el cual considera que "la base de los factores psicosociales reside en la percepciones y experiencias de los sujetos de ciertos aspectos del ambiente laboral que originan trastornos psicológicos, fisiológicos y comportamentales". (García, Castaño \& Fernández, 2007, p.56).

La Organización Mundial de la Salud (OMS, 2004) en su informe, "La organización del trabajo y el estrés", público que un empleado con niveles de estrés considerables, suele no tener motivación, ser enfermizo y por ende ser menos productivo. (p.1).

En una investigación realizada por Marulanda (2007) sobre el estrés laboral, encontró los siguientes resultados que detallaremos a continuación:

El estrés es un estímulo que nos arremete emocional o físicamente, ya que los resultados arrojaron que el 70 u $80 \%$ de los trabajadores de una empresa faltan a su centro laboral por temas de estrés (aunque se disfracen de enfermedades u otras causas), esto según investigaciones realizadas en Latinoamérica.

El Desempeño laboral bajo de un trabajador, nos dice que algo no está funcionando bien en las organizaciones; es allí 
donde las empresas deben tomar un rol protagónico para evitar este tipo de enfermedades, sobre todo para evitar que las operaciones y el rendimiento de los trabajadores no se ven afectadas.

Como respuesta a esta problemática que afecta a nivel mundial, algunas entidades gubernamentales y organizaciones internacionales como la Unión Europea y la Organización Mundial de la Salud han insistido en implementar estrategias para la prevención y el control del estrés, muchos son los que padecen estrés laboral y nunca han sido asistidos por las entidades promotoras de salud. (Marulanda, 2007, p.12).

En una investigación realizada sobre el estrés se hizo comparaciones para saber su incidencia en las personas con distintas edades, género y actividad económica, un ejemplo de esto es la de Estrés en América que evaluó cuales eran los grupos con mayores niveles de estrés; las mujeres, los jóvenes y los padres, arrojo datos como que un $51 \%$ de las mujeres han permanecido despiertas por la noches debido a situaciones de estrés en contraste con un 32\% en los hombres, los padres son los adultos más estresados y en consecuencia han presentado problemas de salud física y emocional, algunos de los síntomas que manifestaron son irritabilidad con un $44 \%$, ansiedad $43 \%$, sentirse abrumado $42 \%$ e ira en un 35\%. Los adultos jóvenes presentan mayores niveles de estrés que otras generaciones, esto relacionado a que no hay buenas estrategias de afrontamiento, sienten sensaciones de soledad y asilamiento (Asociación Americana de Psicología [APA], 2007)

\section{OBJETIVO DEL ENSAYO}

En este artículo de reflexión, pretendo dar a conocer como el estrés laboral incide en el desempeño de los trabajadores dentro de las organizaciones; de una manera didáctica y sintetizada. Primeramente, vamos a definiendo cada variable y seguidamente mostraremos algunos modelos explicativos más resaltantes del estrés en el ámbito empresarial; así mismo mostraremos algunos estresores y enfermedades que puedan generar estrés, y para concluir brindaremos algunas pautas para el diseño de los programas de intervención para un mejor manejo del estrés dentro de las empresas.

\section{ARGUMENTACIÓN}

El estrés Laboral se puede definir como las nocivas reacciones físicas y emocionales, que se da cuando las exigencias del trabajo se exceden. Muchas veces este concepto "se confunde con el desafío", pero no es lo mismo. Es un tipo de estrés asociado al ámbito del trabajo que puede ser puntual o crónico, aunque la mayoría de los casos responde al segundo tipo mencionado (Cavanaugh, Boswell, Roehling y Boudreau, 2000).

Para el autor Galán (2012) la palabra estrés proviene del termino:

Sajón stress que significa tensión, respuesta del individuo cuando se ve expuesto a situaciones que le demanda cambio en su repertorio de conductas cotidianas, porque se puede ver afectada su capacidad de control sobre los acontecimientos o en condiciones más demandantes de su integridad; en estas circunstancias el individuo genera una respuesta a nivel fisiológico que lo preparan para tener un rendimiento superior al normal. (p.120)

De igual forma para el autor Brengelmann (1987) el termino estrés se origina:

de la física y la arquitectura y esto se refiere a la fuerza que se aplica a un objeto, que puede deformarlo o romperlo. En la psicología, estrés suele hacer referencia a situaciones que implican demandas fuertes para el individuo y que pueden agotar sus recursos de afrontamiento. (p.80)

No hay una sola definición, más bien son varias las teorías que han dado una definición al estrés, en esta revisión se nombran las tres principales: "la primera de ellas en el contexto histórico elaborada por Selye, es la teoría basada en la respuesta que explica el estrés como lo que produce un conjunto de respuestas fisiológicas en el individuo llamado Síndrome de Adaptación General" (Floria, 2013).

La definición del término estrés ha sido muy controvertida desde el momento en que se incorporó a la psicología el fisiólogo canadiense Selye (1956) quien definió, al estrés como: "reacción o respuesta del individuo (cambios fisiológicos, reacciones emocionales, cambios 
conductuales, etc.); estimulo (capaz de provocar una reacción de estrés); interacción entre las características del estímulo y recursos del individuo". (Selye, 1956). Siendo la definición más completa la de este autor.

Según Melgosa (1999) el estrés tiene dos componentes básicos: "los agentes estresores o estresantes, y la respuesta del estrés". (p.54).

Asimismo, es importante poner enfasis en el desempeño laboral ya que; la productividad y permanencia dentro de mercado de una empresa u organización, depende mucho de un buen desempeño de los trabajaores. Porque esta demostrado por varios teoricos, que a mayor bienestar del trabajador mayor rendimiento, y mayor productividad.

\section{Causas del Estrés.}

Si bien es cierto, el estrés se manifiesta como una respuesta normal del organismo ante un evento de riesgo o peligro; nuestro organismo se acomoda para hacerle frente o huir mediante: "la secreción de sustancias como la adrenalina, producida principalmente en unas glándulas llamadas "suprarrenales" o "adrenales". La adrenalina se disemina por toda la sangre y es percibida por receptores especiales en distintos lugares del organismo, que responden para prepararse para la acción".

A continuación, mostrare la figura 1 de los factores y síntomas que provoca esta enfermedad.

El medico Canadiense Hans Seyle (1926) identifico, que el estrés tiene 3 fases para explicar su aparición y estas son (Ver figura 2):

Fase de alarma. Ante la percepción de una posible situación de estrés, el organismo empieza a desarrollar una serie de alteraciones de orden fisiológico y psicológico (ansiedad, inquietud, etc.) que lo predisponen para enfrentarse a la situación estresante.

Fase de resistencia. Supone la fase de adaptación a la situación estresante. En ella se desarrollan un conjunto de procesos fisiológicos, cognitivos, emocionales y comportamentales destinados a "negociar" la situación de estrés de la manera menos lesiva para la persona.

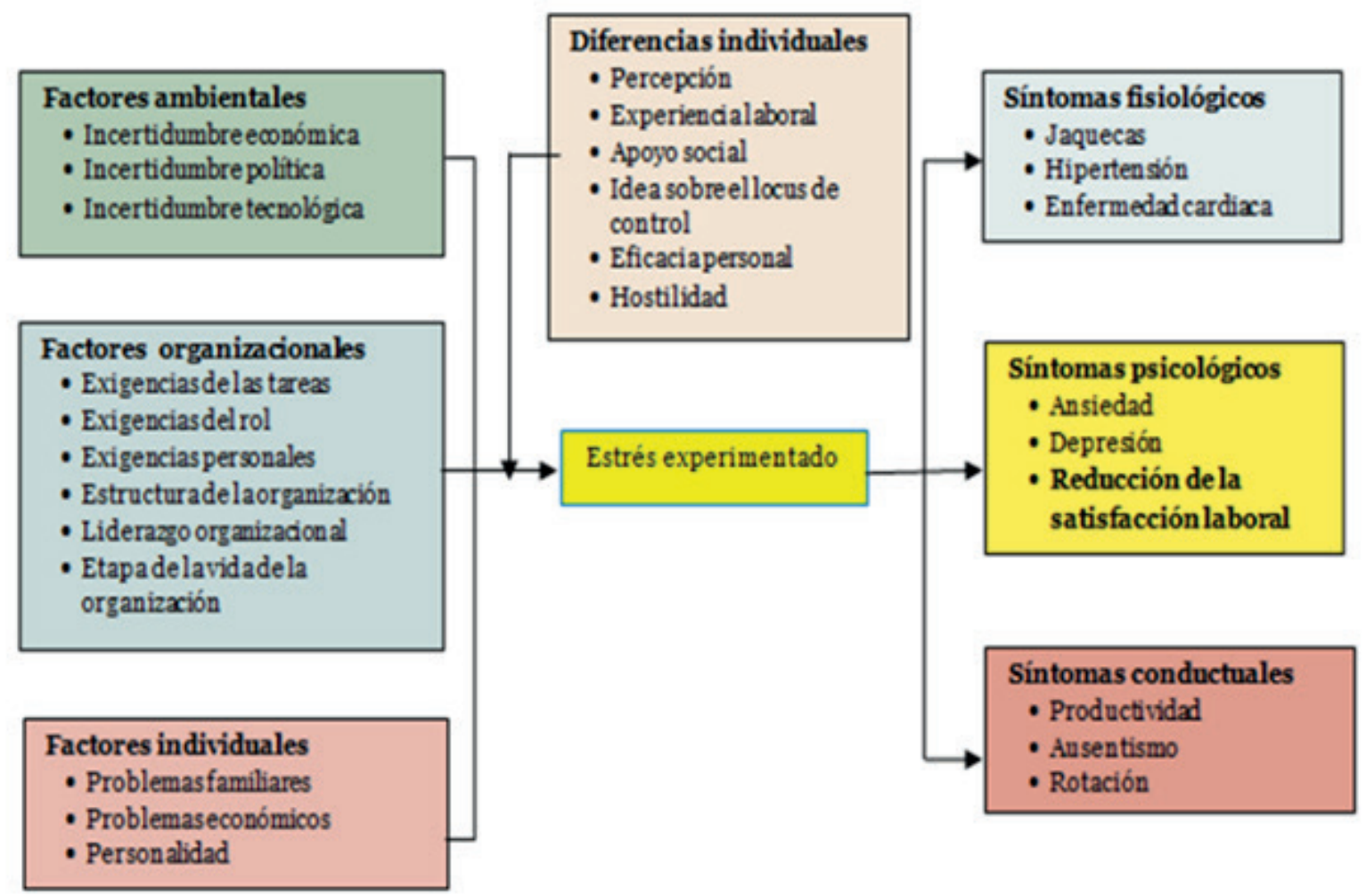

Figura 01. Qué ocasiona el estrés. Tomado de http://www.psicomundo.com/foros/trabajo/estres.htm 


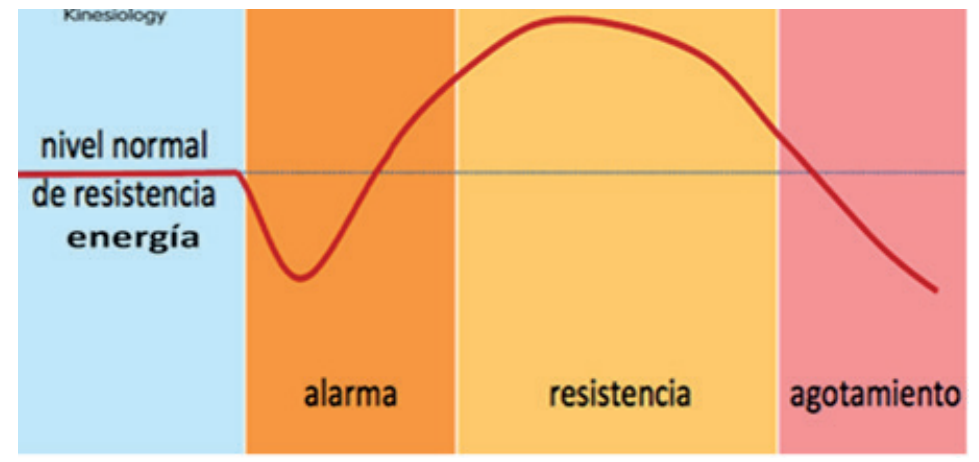

Figura 02. Fases del Estrés. Fuente Dr. Seyle. "Las fases del estrés".

Fase de agotamiento. Si la fase de resistencia fracasa, es decir, si los mecanismos de adaptación ambiental no resultan eficientes se entra en la fase de agotamiento donde los trastornos fisiológicos, psicológicos o psicosociales tienden a ser crónicos o irreversibles. (Seyle, 1926).

Para el autor Peiró (1992), los estresores están clasificadas de la siguiente manera:

Estresores del ambiente físico: Ruido, vibración, iluminación, etc.

Demandas estresantes del trabajo: Turnos, sobrecarga, exposición a riesgos.

Contenidos del trabajo: Oportunidad de control, uso, habilidades, variedad de tareas, feedback, identidad de tarea, complejidad del trabajo.

Estrés por desempeño de roles: Conflicto, ambigüedad y sobrecarga.

Relaciones interpersonales y grupales: Superiores, compañeros, subordinados, clientes.

Desarrollo de carrera: Inseguridad en el trabajo, transiciones, estresores en diferentes estadios.

Nuevas tecnologías: Aspectos ergonómicos, demandas, adaptación a cambios, implantación.

Estructura organizacional.

Clima organizacional.

Estrés por la relación trabajo y otros ámbitos de la vida (familia, etc.): Parejas en las que los dos trabaja. (Peiró, 1992. p. 19).
Cualquier suceso que genere una respuesta emocional, puede causar estrés. Esto incluye tanto situaciones positivas (el nacimiento de un hijo, matrimonio) como negativas (pérdida del empleo, muerte de un familiar).

\section{Signos y Síntomas.}

El estrés laboral puede causar muchos síntomas, y en paralelo va afectando al organismo. entre los síntomas más comunes podemos enumerar: "a la depresión o ansiedad, dolores de cabeza insomnio, indigestión, sarpullidos, disfunción sexual, palpitaciones rápidas, nerviosismo, hasta diabetes".

\section{Tratamiento.}

Los expertos recomiendan prevenir o tratar de reducir el estrés lo máximo posible, antes que produzca daños severos. Se sugiere algún tipo de psicoterapia, tranquilizantes, antidepresivos. En el ámbito organizacional hay varias formas de enfrentar esta problemática, pero para ello los empresarios deben primero tomar conciencia que existe esta enfermedad, que esta aqueja a los trabajadores de su empresa, y manifestándose con un bajo desempeño. Una manera de intervenir seria la prevención primaria, focalizada en intervenir directamente en la organización y sus recursos disponibles, así mismo la intervención secundaria es una segunda opción que consiste en trabajar sobre las personas directamente afectadas para dotarlas de estrategias de afrontamiento y prevenir en ellas el estrés.

A través de diversos métodos se puede dotar al trabajador de recursos de afrontamiento del estrés laboral cuando las medidas organizativas no dan resultados. Estas técnicas pueden ser: 
- Técnicas generales: Tener una dieta adecuada, distraerse y realizar actividades entretenidas.

- Técnicas cognitivo-conductuales: Restructuración cognitiva, inoculación de estrés, detección de pensamiento, y otras técnicas más.

- Técnica de relajación: Relajación muscular, antogena, y el control de la respiración.

\section{Consejos para afrontar el Estrés.}

A continuación, enumeraremos algunos consejos para poder lidiar y prevenir el estrés en nuestra vida cotidiana:

- Identificar nuestras prioridades.

- Relacionarse con personas positivas, que puedan brindar apoyo emocional.

- Disfrutar de las cosas que hacemos, dándonos un tiempo necesario en cada actividad realizada.

- Evitar pensar obsesivamente sobre los problemas que puedan existir.

- Realizar ejercicios de manera cotidiana.

\section{CONCLUSIONES}

En el presente ensayo se ha intentado dar una visión general de la problemática que asecha a las organizaciones hoy en día, diversos estudios realizados sobre el estrés y su incidencia en el desempeño laboral, lo confirman. Las investigaciones se han dado en diversas poblaciones tales como: en médicos, en profesores, estudiantes, madres de familia, administradores, y otros.

Se considera que el estrés laboral es un detonante para dar inicio a muchas enfermedades con diversos síntomas como: "los trastornos digestivos, falta de sueño, dolor de cabeza y tensiones musculares que van deterioran la calidad de vida de las personas".

Por ello se recomienda, su pronta intervención porque no solo ayudaría a las organizaciones a prevenir enfermedades futuras; sino que también con ello se mejoraría el desempeño laboral de los trabajadores, se reduce el ausentismo laboral, mejora el sentido de pertenencia, mejora la satisfechos, incrementaría la productividad y proactividad.

En generales, hay muchos estudios realizados sobre la variable "estrés laboral" y en su gran mayoría coinciden que un alto grado de estrés puede generar diversas enfermedades, y un bajo desempeño laboral en trabajadores de empresas u organizaciones. Los conocimientos que nos dejan las investigaciones sobre las causas, sintomatología y consecuencias podemos usarlo de forma proactiva; con la finalidad de prevenir e intervenir de manera oportuna para evitar que el estrés laboral cause daños irreversibles tanto en nuestra salud, física y psicológica.

\section{AGRADECIMIENTOS Y RECONOCIMIENTOS}

A mis hijos por ser mi norte y mi sostén emocional hacía el avance de mi desarrollo profesional, y sobre todo por ser los principales impulsores de mis sueños, a la Universidad Nacional Mayor De San Marcos por permitirme lograr este desarrollo, y mi asesor de tesis por creer en mí.

\section{REFERENCIA BIBLIOGRÁFICA}

Almirall, H. (1996). Análisis Psicológico del Trabajo.

American Psychological Association. (2014). Stress in América. http://www.apa.org/news/press/ releases/stress/2014/stress-report.pdf

Brengelmann, J. (1986). Estrés, superación y calidad de vida en personas sanas y enfermas. Evaluación Psicológica, 2, 47-78.

Campos, M. A. Causas y efectos del estrés laboral. San Salvador: Universidad de El Salvador, Escuela de Ingeniería Química. (2006).

Cavanaugh, M., Boswell, W., Roehling, M., y Boudreau, J. (2000). An empirical examination of self-reported work stress among US managers. Journal of applied psychology, 85(1), 65.

Keith, D. (1994). Comportamiento Humano en el Trabajo.

Marulanda, I. (2007). Estrés laboral enemigo silencioso de la salud mental y la satisfacción con la vida. Bogotá: Uniandes.

Peiró, J. M., Salvador, A. Desencadenantes del Estrés Laboral (1ª. ed.). España: Editorial UDEMA S. A. (1992).

Sánchez, S., y Pongo, O. (2014). Tendencias Contemporáneas. Metodología y Estadística. 1ae edición. Imprenta UNFV. 